\title{
COMPOSTAJE DE BIOSÓLIDOS DE PLANTAS DE TRATAMIENTO DE ÁGUAS RESIDUALES
}

\author{
PATRICIA TORRES ${ }^{1}$, ANDREA PÉREZ ${ }^{2}$, JUAN C. ESCOBAR ${ }^{3}$ \\ IRIS E. URIBE ${ }^{4}$, RICARDO IMERY ${ }^{5}$
}

RESUMO: Foi avaliada a compostagem dos biossólidos gerados na Estação de Tratamento de Esgotos - ETE, de Cañaveralejo, da cidade de Cali - Colômbia. Ainda que o processo se mostrasse viável, a incorporação de materiais de suporte e emenda foi favorável ao mesmo e à qualidade do produto final ao melhorar as condições de manejo, estrutura e porosidade do biossólido (B), além de melhorar as relações carbono/nitrogênio. Dos materiais avaliados, os que apresentaram melhor desempenho como materiais de suporte (MS) e Emenda (ME), foram os resíduos de poda e a cachaça, respectivamente; a relação ótima B:MS:ME, em percentagem, foi 72:10:18. Do ponto de vista da gestão dos resíduos e considerando o crescente incremento no número de ETEs municipais, este estudo mostrou que o composto produzido a partir de biossólidos pode ser considerado um material com potencial agrícola; adicionalmente, nos casos em que a única opção é a disposição final, o processo permitiu reduzir o volume a ser disposto até em $70 \%$.

PALAVRAS-CHAVE: compostagem, gestão de biossólidos, materiais de emenda e suporte.

\section{PLANT BIOSOLIDS COMPOSTING OF WASTEWATER TREATMENT}

\begin{abstract}
It was evaluated the bio solid composting in Cañaveralejo Wastewater Treatment Plant in Cali - Colombia. Although the process was viable, the bulking agent (BA) incorporation improved the final product $\mathrm{C} / \mathrm{N}$ relation. Municipal yard trimmings and sugar cane waste were the best bulking agent and amendment material, respectively. The B:BA:AM optimal relation, on percent, was 72:10:18. In terms of waste management and considering the increasing on the municipal wastewater treatment plants number, this study showed that the bio solid compost could be considered as a material with agricultural potential use; additionally when the option is just the bio solid final disposition, the composting process can reduce its disposal volume at $70 \%$.
\end{abstract}

KEYWORDS: amendment and bulking agents, biosolids management, composting.

\section{INTRODUCCIÓN}

Los biosólidos son lodos de Plantas de Tratamiento de Aguas Residuales - PTAR que pueden ser empleados benéficamente después de someterse a procesos de estabilización (WEF, 1998). La PTAR Cañaveralejo de la ciudad de Cali, Colombia, tratando un caudal de $3.5 \mathrm{~m}^{3} \mathrm{~s}^{-1}$ puede generar entre 60 y $80 \mathrm{t} \mathrm{dia}^{-1}$ de biosólidos, dependiendo de la modalidad de operación: Tratamiento Primario Convencional o Tratamiento Primario Avanzado - TPA (EMCALI, 2002).

El compostaje es un proceso biológico exotérmico de conversión de la materia orgánica presente en los residuos hacia formas más estables como el humus, la cual es realizada por microorganismos como bacterias, hongos y actinomicetos que requieren de ciertas condiciones ambientales controladas que faciliten el incremento de la temperatura (usualmente entre $55-60{ }^{\circ} \mathrm{C}$ ) para la destrucción de patógenos (KIELY, 1999). El compostaje de biosólidos garantiza un

\footnotetext{
${ }^{1}$ Ingeniera Sanitaria, PhD, Profesora Asociada. Escuela EIDENAR, Facultad de Ingeniería, Universidad del Valle, Cali - Colombia, patoloz@univalle.edu.co

${ }^{2}$ Ingeniera Sanitaria, Asistente de Investigación, Escuela EIDENAR, Facultad de Ingeniería, Universidad del Valle, Cali Colombia, andreaperezvidal@ hotmail.com

${ }^{3}$ Ingeniero Sanitario, PhD, Empresas Municipales de Cali, EMCALI, jcescobar@emcali.com

${ }^{4}$ Ingeniera Sanitaria, M.Sc. ,Ingeniería Sanitaria y Ambiental, Universidad del Valle, iriseuge@ hotmail.com

${ }^{5}$ Ingeniero Sanitario, M.Sc., Ingeniería Sanitaria y Ambiental, Universidad del Valle, rimery@ telesat.com.co

Recebido pelo Conselho Editorial em: 23-11-2005
}

Aprovado pelo Conselho Editorial em: 20-12-2006 
producto con $\mathrm{pH}$ entre 6,5 y 8,0 unidades que favorece el crecimiento de las plantas, reduce la movilidad de metales pesados (EPA, 1999) y puede ser usado benéficamente como acondicionador de suelos (KUTER et al., 1995).

Los biosólidos generados en PTAR presentan tendencia a la compactación y baja porosidad que ocasionan dificultades durante el proceso de compostaje por una inadecuada aireación, lo que se puede corregir adicionando Materiales de Soporte para mejorar la porosidad y estructura de las pilas de compost y garantizar el ingreso del oxígeno necesario para favorecer las condiciones aerobias del proceso (KUTER et al., 1995).

Los materiales de soporte son variados: vegetales leñosos como residuos de poda de zonas verdes o de jardinería, aserrín, paja, materiales previamente compostados (EPA, 1999), trozos de neumáticos, astillas de pino, viruta de madera y desechos agrícolas (URIBE, 2005). Algunos materiales de soporte actúan también como enmienda, al contribuir al mejoramiento de las características químicas del producto final, como es el caso de la cascarilla de arroz que, además de mejorar la estructura de la pila, aporta potasio (WEF, 1998).

Los Materiales de Enmienda son fuente de energía y carbono y suministran nutrientes rápidamente disponibles mejorando y ajustando además el contenido de humedad de la pila (KUTER et al., 1995). Los biosólidos son residuos ricos en nitrógeno con relaciones C/N entre 5,0 y 11,0; los materiales de enmienda, ricos en carbono, permiten ajustar esta relación a los valores recomendados para garantizar la eficiencia del proceso (20 a 30) (FERNANDES \& PEREIRA DA SILVA, 1999).

Algunos materiales utilizados son: residuos orgánicos municipales, paja, bagazo y cachaza de caña (residuos de la industria del azúcar) (PROSAB, 1999), materiales minerales como la fosforita (IMERY, 2005), viruta de madera, tallos de maíz, ceniza, pulpa de remolacha (KUTER et al., 1995).

En esta investigación se evaluó la viabilidad del proceso de compostaje de biosólidos de la PTAR-C solo y mezclado con Materiales de Soporte (residuo de poda y viruta de madera) y Materiales de Enmienda (cachaza y residuos orgánicos de plazas de mercado - ROPM) para evaluar su efecto sobre el mejoramiento del proceso y la calidad del producto final obtenido.

\section{MATERIALES Y MÉTODOS}

El estudio fue desarrollado en una planta piloto de compostaje con un área de $468 \mathrm{~m}^{2}$, distribuidos en las zonas de mezcla y adecuación (20\%), digestión (60\%) y enfriamiento y maduración (20\%). Para la primera y tercera zonas, se adecuó el techo con una cubierta de placas onduladas en fibrocemento que impiden el paso del sol y en la segunda zona se instaló un plástico pigmentado tipo invernadero calibre 7 para permitir el paso de la luz solar y lograr el efecto invernadero que favorece la digestión.

La fase experimental se dividió en dos etapas: Selección del Material de Soporte y Selección de Material de Enmienda. Los criterios para la selección de los materiales fueron: $i$ ) alta disponibilidad regional, ii) accesibilidad al material sin costo de adquisición, iii) apertura interinstitucional a la suscripción de convenios, iv) estructura y tamaño de la partícula, $v$ ) resistencia del material de soporte a la biodegradación y vi) contenido de nutrientes del material de enmienda (TORRES et al., 2004). Al inicio de cada etapa se caracterizaron todos los materiales empleados para el compostaje midiendo las variables mostradas en la Tabla 1. 
TABLA 1. Caracterización inicial de los materiales empleados para el compostaje.

\begin{tabular}{lc}
\hline Variable* & Método de Medición* \\
\hline Fisicoquímicas & $\begin{array}{c}\text { Potenciométrico } \\
\text { Gravimétrico }\end{array}$ \\
\hline pH (unidades) & $\begin{array}{c}\text { Digestión y Titulación } \\
\text { Humedad (\%) }\end{array}$ \\
Fósforo y potasio (\%) & $\begin{array}{c}\text { Atómica } \\
\text { Microbiológicas y parasitológicas }\end{array}$ \\
\hline Coliformes totales y fecales (UFC/g) & Filtración por membrana \\
Huevos de helmintos viables $\left(\mathrm{N}^{\circ} \mathrm{HH} / \mathrm{g}\right)$ & Bailenger modificado
\end{tabular}

\section{Etapa 1. Selección del material de soporte}

Se evaluaron los residuos de poda de zonas verdes de la ciudad de Cali (P) y la viruta de madera (V) procedente de una industria papelera de la región. Las pilas de compost fueron conformadas con Biosólidos (B), Material de Soporte (MS) y Material de Enmienda (residuos orgánicos de plazas de mercado-ROPM). En todos los ensayos, se garantizó una relación fija B:ROPM de 60:40 y se varió la proporción de material de soporte.

\section{Ensayo 1}

\section{Selección del porcentaje óptimo}

Para cada material de soporte, se evaluaron tres porcentajes en peso: $5 \% ; 10 \%$ y $20 \%$. Se conformaron 5 pilas de 1,0 tonelada por duplicado con las características mostradas en la Tabla 2.

TABLA 2. Composición de las pilas para selección del porcentaje óptimo de material de soporte.

\begin{tabular}{cc}
\hline Pila & Conformación \\
\cline { 2 - 2 } & B : ME: MS \\
\hline Testigo 1 & $100: 0: 0$ \\
Testigo 2 & $60: 40: 0$ \\
$5 \%$ MS & $57: 38: 5$ \\
10\% MS & $54: 36: 10$ \\
20\% MS & $48: 32: 20$ \\
\hline
\end{tabular}

MS: Material de soporte (residuo de poda - P o viruta de madera - V) ; ME: material de enmienda.

Las variables de seguimiento del proceso se muestran en la Tabla 3 y las variables de respuesta, medidas al inicio y al final del proceso, corresponden a las mostradas en la Tabla 1 con excepción del pH y la humedad. El material compostado fue cribado con un tamiz de $10 \mathrm{~mm}$, con el fin de separar el material de soporte y retirar los grumos formados.

TABLA 3. Variables de seguimiento del proceso de compostaje.

\begin{tabular}{lcc}
\hline Variable & Método de Medición & Frecuencia \\
\hline Temperatura & Directo con Termómetro de Bulbo 60cm & Diaria \\
$\mathrm{pH}$ & Potenciométrico & 3 veces/ semana \\
Humedad & Gravimétrico & 3 veces/ semana \\
Volteo & Manual & 2 veces / semana* \\
Riego & Manual & Durante el volteo si la humedad $<45 \%$ \\
\hline
\end{tabular}

*La frecuencia dependió de la evolución del proceso 


\section{Ensayo 2}

\section{Selección del material}

A partir del Ensayo 1 se eligió un porcentaje fijo de ambos materiales de soporte y se conformaron 3 pilas de 1.5 tonelada por duplicado, la primera estuvo conformada solo por Biosólido (Testigo 1) y las dos restantes por la combinación B.ME:MS en una proporción 54:36:10. El proceso fue monitoreado del mismo modo que el Ensayo 1.

\section{Etapa 2. Selección de material de enmienda}

El material de soporte fue el seleccionado en la etapa anterior - Residuo de poda y se evaluaron dos materiales de enmienda: Cachaza procedente de un ingenio azucarero y Residuos Orgánicos de Plazas de Mercado - ROPM, en dos porcentajes cada uno como lo muestra la Tabla 4. La pila Testigo estuvo constituida solo por biosólido. Todas las pilas fueron conformadas por duplicado, con un peso total de 1.0 tonelada.

TABLA 4. Composición de las pilas para selección del material de enmienda.

\begin{tabular}{cc}
\hline Pila & Conformación \\
\cline { 2 - 2 } & B :ME:MS \\
\hline Testigo & $100: 0: 0$ \\
$18 \% \mathrm{ME}$ & $72: 18: 10$ \\
$36 \% \mathrm{ME}$ & $54: 36: 10$ \\
\hline
\end{tabular}

MS: Material de Soporte - residuo de poda

ME: Material de Enmienda - cachaza y residuos orgánicos de plazas de mercado - ROPM.

Para evitar la formación de grumos y compactación de las pilas, el orden de mezcla de los materiales fue la incorporación al biosólido inicialmente del material de soporte y posteriormente del material de enmienda. El seguimiento del proceso fue similar al de la etapa 1, ampliando la frecuencia de medición de las variables de respuesta. Se utilizaron tamices de $10 \mathrm{~mm}$ para separar el material de soporte y de $5 \mathrm{~mm}$ para mejorar las características estéticas del producto final.

El análisis de las variables de seguimiento se realizó con base en estadística descriptiva y la construcción de gráficos que permitieron observar la evolución del proceso. Para la variable temperatura se emplearon análisis de regresión o líneas de tendencia que se ajustaron a una polinomial de orden 6 y para evaluar el comportamiento de las diversas variables de respuesta se adoptó el diseño de bloques completos al azar.

\section{RESULTADOS Y DISCUSIÓN}

\section{Etapa 1. Selección de material de soporte}

\section{Características iniciales}

La Tabla 5 presenta las características de los materiales empleados en los estudios para selección del material de soporte.

En la tabla se observa que los valores de $\mathrm{pH}$ para todos los materiales se encuentran dentro del intervalo óptimo recomendado para el proceso de compostaje, entre 6,5 - 8,0 unidades (KIELY, 1999). El rango ideal del porcentaje de humedad es de 30 a 60\% (KUTER et al., 1995 y ABAD et al., 1996), lo que indica que hay un exceso de humedad en el Biosólido y el ROPM que puede ser contrarrestado incorporando materiales de soporte dado su bajo contenido de humedad.

La baja relación $\mathrm{C} / \mathrm{N}$ característica de los biosólidos, se puede mejorar con la incorporación de los materiales de soporte y de enmienda; en los materiales de soporte, la alta relación se debe a la presencia de compuestos de difícil biodegradabilidad como la lignina y la celulosa presentes en la viruta y las ramas y hojas secas respectivamente. Los valores de las variables microbiológicas y parasitológicas indican contaminación en todos los materiales, principalmente en el biosólido; el 
compostaje se ha mostrado como una potencial alternativa de tratamiento para la reducción de la carga microbiológica contaminante.

TABLA 5. Características de los materiales de partida.

\begin{tabular}{|c|c|c|c|c|}
\hline \multirow{2}{*}{ Variables* } & \multirow{2}{*}{ Biosólido } & \multirow{2}{*}{$\frac{\text { Material de Enmienda }}{\text { ROPM }^{* *}}$} & \multicolumn{2}{|c|}{ Material de Soporte } \\
\hline & & & Poda & Viruta \\
\hline \multicolumn{5}{|l|}{ Fisicoquímicas } \\
\hline pH (Unidades) & 6,7 & 6,9 & 7,3 & 6,8 \\
\hline Humedad $(\%)$ & 67,9 & 71,3 & 18,9 & 31,1 \\
\hline Carbono orgánico $(\%)$ & 18,6 & 19,5 & 28,3 & 13,7 \\
\hline Nitrógeno total - NTK (\%) & 2,73 & 1,47 & 1,33 & 0,41 \\
\hline Relación C/N & 6,8 & 13,3 & 21,3 & 33,4 \\
\hline Fósforo (\%) & 1,35 & 0,28 & 0,15 & 0,04 \\
\hline Potasio $(\%)$ & 0,18 & 1,21 & 0,97 & 0,17 \\
\hline \multicolumn{5}{|c|}{ Microbiológicas y parasitológicas } \\
\hline Coliformes totales (UFC/g) & $1,310^{7}$ & $1,1410^{7}$ & $8,610^{6}$ & $8,8 \quad 10^{6}$ \\
\hline Coliformes fecales (UFC/g) & $1,0 \quad 10^{7}$ & $7,2 \quad 10^{6}$ & $2,0 \quad 10^{6}$ & $2,2 \quad 10^{6}$ \\
\hline Huevos de helminto viables/g & 8 & 3 & 3 & 2 \\
\hline
\end{tabular}

\section{Ensayo 1}

\section{Selección del porcentaje óptimo}

En las Figuras 1a y $1 \mathrm{~b}$ se presentan las líneas de tendencia promedio de la temperatura durante el proceso de compostaje para las pilas con residuo de poda y viruta de madera respectivamente.

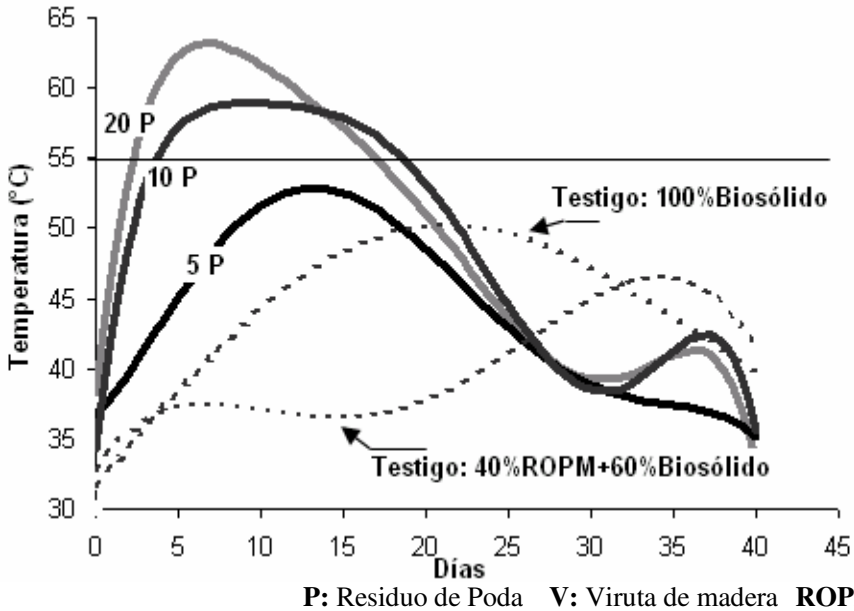

a)

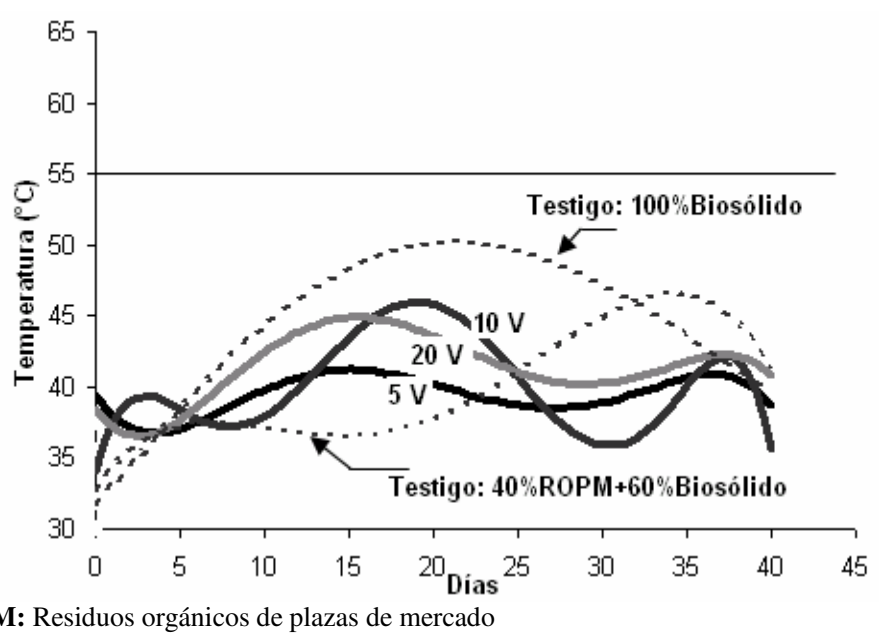

b)

FIGURA 1. Comportamiento de la temperatura. a) Pilas con residuo de poda y b) Pilas con viruta.

Para una adecuada higienización del compost, se requieren periodos entre 4 y 15 días con temperaturas superiores a $55^{\circ} \mathrm{C}$ (HOOG et al., 2002) o una hora por encima de $70{ }^{\circ} \mathrm{C}$ (KIELY, 1999). El Testigo 1 alcanzó temperaturas por encima de los $55^{\circ} \mathrm{C}$ solo durante 3 días y el Testigo 2 alcanzó una temperatura máxima de $45^{\circ} \mathrm{C}$, lo que pudo deberse al exceso de humedad que ocasionó la formación de grumos que no pudieron destruirse a pesar de los volteos. Esta situación reafirma la necesidad de incorporar un material de soporte que aporte estructura y mejore la aireación de la pila durante el proceso. 
Las pilas en las que se incorporó el residuo de poda mostraron un mejor comportamiento con relación a las pilas Testigo, presentando una etapa mesofílica de menor duración. Los porcentajes de $10 \%$ y $20 \%$ favorecieron la permanencia de temperaturas por encima de $55^{\circ} \mathrm{C}$ durante casi dos semanas (12 días y 14 días, respectivamente). En las pilas en que se empleó la viruta, independiente del porcentaje utilizado, no se alcanzaron las temperaturas recomendadas debido probablemente al tamaño de la viruta (mayor a $5 \mathrm{~cm}$.) que impidió una adecuada aireación de la pila. Debido a que con ambos materiales de soporte no se hallaron diferencias estadísticas significativas entre el 10 y el $20 \%$ y que el objetivo de la investigación fue comportar el mayor porcentaje de biosólido, se seleccionó el $10 \%$ de material de soporte como el valor más adecuado.

\section{Ensayo 2}

\section{Selección del Mejor Material de Soporte}

La Figura 2 muestra el comportamiento de la temperatura durante el proceso de compostaje de la pila testigo y las conformadas con la combinación B.ME:MS en una proporción 54:36:10. En ellas se observa que el uso de los dos materiales de soporte favoreció el proceso de compostaje acelerando el inicio de la etapa termofílica y garantizando una mayor permanencia de altas temperaturas.

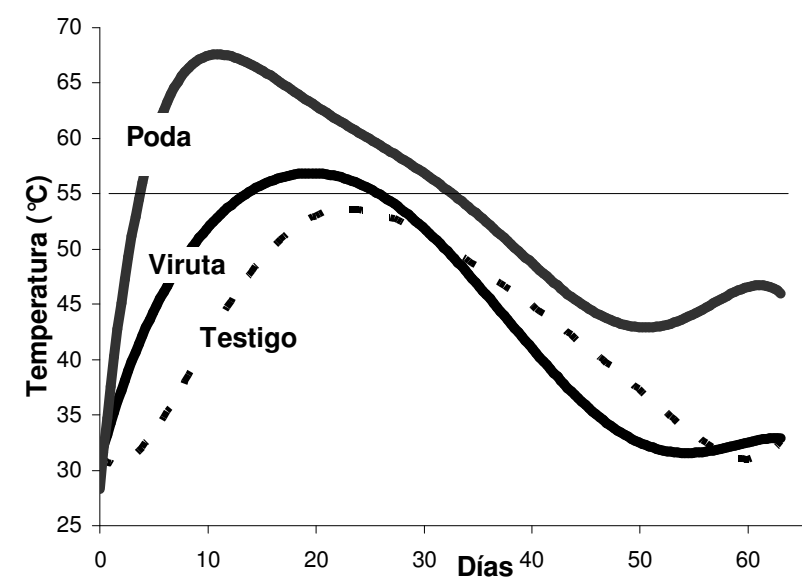

FIGURA 2. Comportamiento de la temperatura a lo largo del proceso de compostaje.

Comparando el comportamiento de las pilas con material de soporte, estadísticamente se verificó que la pila en la que se incorporó la poda, presentó el mejor desempeño tanto desde el punto de vista del rápido inicio de la etapa termofílica como del mayor período de tiempo con temperaturas superiores a $55^{\circ} \mathrm{C}$ ( 28 días).

\section{ETAPA 2. Selección material de enmienda}

\section{Características iniciales}

La Tabla 6 muestra las características iniciales de los materiales usados, en ella se observa que el $\mathrm{pH}$ en todos los materiales se encuentra dentro del intervalo recomendado para lograr un óptimo proceso de compostaje. La humedad es similar en los materiales de enmienda y en el biosólido, lo que indica que todos los materiales requieren la incorporación de material de soporte para ajustarla. Aunque los materiales de enmiendan presentan una relación $\mathrm{C} / \mathrm{N}$ mayor que la del biosólido, no alcanzan el rango recomendado entre 20-30 (SANEPAR, 1999). Desde el punto de vista microbiológico y parasitológico todos los materiales presentan contaminación. 
TABLA 6. Características de los materiales de partida.

\begin{tabular}{|c|c|c|c|}
\hline \multirow{2}{*}{ Variables* } & \multirow{2}{*}{ Biosólido } & \multicolumn{2}{|c|}{ Material de Enmienda } \\
\hline & & Cachaza & $\mathrm{ROPM}^{* *}$ \\
\hline \multicolumn{4}{|l|}{ Fisico-químicas } \\
\hline pH (Unidades) & 6,7 & 8,0 & 7,40 \\
\hline Humedad (\%) & 69,2 & 72,6 & 71,3 \\
\hline Carbono orgánico $(\%)$ & 16,7 & 17,60 & 13,70 \\
\hline Nitrógeno total - NTK (\%) & 2,37 & 1,22 & 1,39 \\
\hline Relación C/N & 7,05 & 14,43 & 9,86 \\
\hline Potasio $(\%)$ & 0,10 & 0,21 & 0,94 \\
\hline Fósforo (\%) & 0,87 & 1,49 & 0,26 \\
\hline \multicolumn{4}{|l|}{ Microbiológicas y parasitológicas } \\
\hline Coliformes totales (UFC/g) & $4,3 \quad 10^{10}$ & $5,710^{\wedge} 10$ & $1,1410^{\wedge} 7$ \\
\hline Coliformes fecales (UFC/g) & $7,1 \quad 10^{8}$ & $2,510^{\wedge} 9$ & $7,210^{\wedge} 6$ \\
\hline Huevos de helminto/g & 13 & 13 & 3 \\
\hline
\end{tabular}

* Valores reportados en base seca, con excepción del Ph.

** ROPM: residuos orgánicos de plaza de mercado.

\section{Selección del mejor material de enmienda}

En la Figura 3 se observa que ambos materiales de enmienda optimizaron el proceso, permitiendo iniciar la etapa termofílica a partir del tercer día y alcanzar temperaturas superiores a $\operatorname{los} 63^{\circ} \mathrm{C}$.

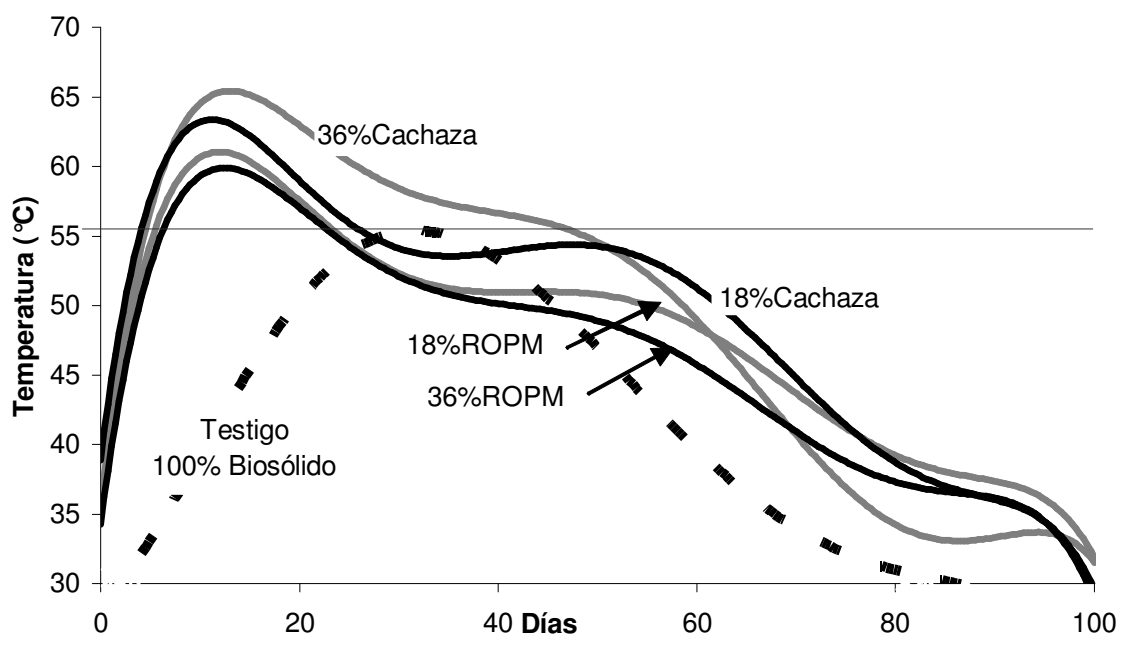

FIGURA 3. Curvas de tendencia de la temperatura durante el proceso de compostaje.

Estadísticamente, se verificó que temperaturas superiores a $55^{\circ} \mathrm{C}$ se mantuvieron por períodos de 15 a 17 días para las pilas con ROPM y entre 32 y 42 días para las pilas con cachaza lo que indica que, de manera general, la cachaza presentó un mejor desempeño. Desde el punto de vista de los dos porcentajes evaluados, no se hallaron diferencias significativas en el comportamiento de las variables de seguimiento y respuesta, por lo cual la proporción más adecuada de material de enmienda para el compostaje de biosólidos fue la del $18 \%$.

Considerando los tipos y proporciones de material de soporte y de enmienda seleccionados para el compostaje de los biosólidos, la pila considerada óptima fue la conformada por $72 \%$ Biosólido, 18\% Cachaza y 10\% Poda. La Tabla 7 muestra la caracterización inicial y final de la pila testigo y la pila óptima; la característica final se compara con algunas referencias bibliográficas y legislaciones internacionales sobre calidad de compost producido con biosólidos. 
TABLA 7. Características iniciales y finales de las pilas testigo y óptima.

\begin{tabular}{lcccccc}
\hline \multirow{2}{*}{ Variable } & \multicolumn{1}{c}{ 100\% Biosólido } & 72\%B $-18 \% \mathrm{C}-10 \% \mathrm{P}$ & \multicolumn{2}{c}{ Valor } & \multirow{2}{*}{ Referencia } \\
\cline { 2 - 6 } & Inicio & Final $(\mathrm{C} 1)$ & Inicio & Final (C2) & Recomendado & \\
\hline pH & 6,70 & 6,50 & 7,05 & 7,40 & $5,0-8,5$ & NCh2880 -2004* \\
Materia orgánica \% & 28,4 & 16,7 & 32,3 & 22,4 & $\geq 25$ & AS4454-1999 \\
Carbono orgánico \% & 16,7 & 9,8 & 19,0 & 13,2 & $>15$ & ICONTEC, 2003 \\
Nitrógeno amoniacal \% & 0,29 & 0,03 & 0,02 & 0,02 & $\leq 0,03$ & NCh2880 -2004* \\
& & & & & & AS4454-1999 \\
Nitrógeno total \% & 2,37 & 1,73 & 1,72 & 1,85 & $>0,8$ & NCh2880 -2004* \\
& & & & & $\leq 25($ Clase A) & AS4454-1999 \\
Relación C/N & 7,1 & 5,6 & 11,1 & 7,1 & $\leq 30$ (Clase B) & NCh280 -2004* \\
Fósforo \% & 0,87 & 1,63 & 0,72 & 2,06 & $\geq 0,1$ & NCh2880 -2004* \\
Potasio \% & 0,10 & 0,08 & 0,19 & 0,37 & 0,025 & SERAPIO, 1996 \\
NPK \% & 3,3 & 3,4 & 2,6 & 4,3 & $\geq 4$ & GÓMEZ, 2000 \\
Densidad real $\left(\mathrm{g} \mathrm{cm}^{-3}\right)$ & - & 2,0 & - & 2,0 & $1,45-2,69$ & ABAD et al., 1996 \\
Densidad aparente $\left(\mathrm{g} \mathrm{cm}^{-3}\right)$ & - & 0,5 & - & 0,4 & $\leq 0,6$ & ICONTEC, 2003 \\
Humedad \% & 70 & 40 & 62 & 35 & $30-45$ & NCh2880 - 2004* \\
\hline
\end{tabular}

*Citado por NAREA, 2004. B: biosólido; C: cachaza; P: residuo de poda

De manera general se observa que el Compost C2 presentó características más próximas a los valores recomendados, principalmente en términos de las variables de interés agronómico materia orgánica, carbono, nitrógeno total y NPK. Los elementos Sodio, Cobre, Manganeso, Boro y Zinc se encontraron entre los límites establecidos por la normatividad para ambos compost; solamente el hierro medido al Compost $\mathrm{C} 1$ presentó un valor de 4,5\%, el cual supera el rango recomendado de 0,3 a 3,0\% (SERAPIO, 1996). Desde el punto de vista parasitológico, no se detectaron huevos de helmintos viables en ninguno de los compost. Aunque la concentración de coliformes totales y fecales se redujo del orden de $10^{10}$ a $10^{7} \mathrm{UFC}^{-1} \mathrm{~g}$ y $10^{8}$ a $10^{4} \mathrm{UFC}^{-1} \mathrm{~g}$ respectivamente, es recomendable considerar un proceso complementario de higienización de este material.

Comparando el peso final e inicial de la pila conformada solo por Biosólido, se alcanzó una reducción aproximada del $70 \%$ lo que ratifica que el compostaje, además de ser una alternativa de estabilización de la materia orgánica (METCALF \& EDDY, 2003) favorece la gestión de estos residuos, disminuyendo los requerimientos de transporte y área para su disposición final.

\section{CONCLUSIONES}

Los resultados del estudio muestran la viabilidad del proceso de compostaje de biosólidos de la PTAR Cañaveralejo de Cali, siendo necesario incorporar materiales de soporte y de enmienda que aporten estructura, manejabilidad y mejoren la relación $\mathrm{C} / \mathrm{N}$ tanto inicial como final. Los materiales que mostraron los mejores resultados fueron el Residuo de Poda y la Cachaza en porcentajes del 10 y $18 \%$ respectivamente.

El proceso de compostaje, además de ser una alternativa de estabilización de la materia orgánica, favorece la gestión de los biosólidos generados en Plantas de Tratamiento de Aguas Residuales, disminuyendo los requerimientos de transporte y área para su disposición final. La reducción en peso de las pilas de biosólido fue del $70 \%$.

Para aumentar el potencial agrícola del compost producidos con biosólidos se recomienda considerar otros materiales que aumenten la relación $\mathrm{C} / \mathrm{N}$ del producto final y evaluar procesos complementarios de higienización del material producido.

\section{AGRADECIMIENTOS}

La investigación fue desarrollada con el apoyo financiero de COLCIENCIAS, el BID, el OCyT de Colombia, EMCALI EICE- ESP y la Universidad del Valle.' 


\section{REFERENCIAS}

ABAD, M.; MARTÍNEZ, P.; MARTÍNEZ, J. Evaluación agronómica de los sustratos de cultivo. Valencia: Departamento de Producción Vegetal, Universidad Politécnica Valencia. 1996.

APHA/AWWA/WPCF. Standard Methods for the examination of water and wastewater. 1998. AS 4454-1999. Australian standard compost, soil conditioners and mulches. Australia, 1999. EMCALI. Estudio sobre Tratamiento Primario Avanzado - TPA. Condiciones de Mezcla y Dosis de Químicos. Planta de Tratamiento de Aguas Residuales de Cañaveralejo, Santiago de Cali, 2002. EPA. ENVIRONMENTAL PROTECTION AGENCY. Biosolids generation, use, and disposal in the United States. Washington, 1999.

FERNANDES, F.; PEREIRA DA SILVA, S. Manual prático para a compostagem de biossólidos. Londrina: Universidade Estadual de Londrina, 1999.

GUTIÉRREZ, H.; DE LA VARA, R. Análisis y Diseño de Experimentos. Mexico: McGraw-Hill Interamericana, 2003. $571 \mathrm{p}$.

GÓMEZ, J. y SÁNCHEZ, M. El proceso de descomposición de residuos vegetales. Palmira: Universidad Nacional de Colombia, 2000. (Cuaderno de Microbiología, 10)

HOOG, D.; BARTH, J.; CENTEMERO, M.; CAIMI, V.; AMLINGER, F.; DEULIEGHER, W.; BRINTON, W.; ANTLER, S. Comparison of compost standards within the EU, North America and Australasia. Banburg, Oxon, 2002. p.41 (Main Report Section 3)

ICONTEC. Norma Técnica Colombiana NTC 5167. Productos para la industria agrícola y materiales usados como fertilizantes y acondicionadores. Colombia, 2003.

IMERY, R. Compostaje de residuos de galería y pulpa de café, Popayán - Cauca. Cali: Universidad del Valle, 2005.

KIELY, G. Ingeniería ambiental: fundamentos, entornos, tecnologías y sistemas de gestión. Mc Graw Hill, 1999. 1.331 p.

KUTER, G.; BLACKWOOD, K.; L.F. DÍAZ, J.; DONOVAN, D.; DURFEE, E.; EPSTEIN, J.; HAY, M.; LANG, T.; RICHARD, G.; SAVAGE, R.; STRATTON, R.; TARDY, T.; WALSH, C.; WILBER III; WILLIAMS, T. Biosolids Composting. Water Environment Federation,

Washington, 1995. $187 \mathrm{p}$.

METCALF; EDDY. Wastewater Engineering. Treatment and Reuse. Washington: McGraw Hill, 2003.

NAREA, C.G. Norma Chilena NCh 2880/2004. Compost Clasificación y requisitos. Servicio agrícola y ganadero. Departamento de protección de recursos naturales. Proyecto de Agricultura Orgánico. Temuco, 2004.

PROSAB. PROGRAMA DE PESQUISA EM SANEAMENTO BASICO. Uso e manejo do lodo de esgoto na agricultura. São Paulo: ABES, 1999. 97 p.

SANEPAR. Reciclagem de biossólidos. Tranformando problemas em soluções. Edición Ministério de Ciencia y Tecnología, FINEP, CNPQ y CAPES. Brasil, 1999. 300 p.

SERAPIO, F.; BRUZON, C. Curso de abono y sustratos orgánicos. Bogotá: Universidad Nacional de Colombia, 1996.

TORRES, P.; ESCOBAR, J.; PÉREZ, A.; IMERY, R.; URIBE, I. Evaluación de la tecnología de compostaje para el aprovechamiento de los biosólidos generados en la PTAR-Cañaveralejo de EMCALI EICE ESP. Colciencias, Universidad del Valle, EMCALI, OCyT, BID y Fundación Biociudad. Cali, 2004. Informe Final.

URIBE, I. Evaluación del compostaje de biosólidos de la PTAR Cañaveralejo de Cali. Cali: Universidad del Valle, 2005.

WEF. WATER ENVIRONMENT FOUNDATION. Biosolids fact. Sheets. USA, 1998. 1 CDROM. 